\title{
Reliability Research of Concrete Pump Main Oil Hydraulic System Based on T-S Fuzzy Fault Tree
}

\author{
Qing Zhao ${ }^{1}$, Lijun $\mathrm{Li}^{1}$, Hongbin Tang ${ }^{2}$ \\ ${ }^{1}$ School of Mechanical and Electronic, Central south University of Forestry and Technology, \\ Changsha, 410004, Hunan, China \\ ${ }^{2}$ College of Automative and Mechanical Engineering Changsha University of Scicence and \\ Technology, Changsha, 410114, Hunan, China
}

Keywords: T-S fuzzy fault tree; Hydraulic system; Fault diagnosis; Reliability analysis.

\begin{abstract}
For improving the reliability of Concrete pump, fault diagnosis is proposed based on the main oil hydraulic pumping system T-S fuzzy fault tree method. The proposed method used fuzzy numbers to describe the probability of failure of hydraulic components and systems to address the failure probability of uncertainty; described by T-S fuzzy door contact between events, to solve the ambiguity and failure mechanisms associated events; fuzzy numbers description of the problem severity, considering the effect of the degree of fault on the system. The method is applied to the fault diagnosis of pumping concrete main oil pump hydraulic system, and achieved good results.
\end{abstract}

\section{Introduction}

Fault tree analysis is a kind of analysis method which makes the cause of the system fault from the whole to the part of the step by step, which can be used for qualitative analysis and quantitative calculation. In the design stage, it can help the potential fault. In the use stage, Can be fault diagnosis, in order to improve the system design to provide quantitative basis.

Fault tree analysis is an important tool for reliability research, which can be used to find the weak links, and provide the basis for the design. The fuzzy logic and TS fuzzy model are introduced into the fault tree analysis, so that the fault tree has the ability to deal with fuzzy information, TS fuzzy fault In the tree analysis, it is not necessary to know the fault probability of the parts accurately, and it is not necessary to know the mechanism of the fault accurately, so as to solve the problem of the uncertainty of the system and the fault mechanism. The importance degree is an important index of the quantitative analysis of the fault tree. When a component fails, it contributes not only to the reliability analysis of the system but also to the optimal design and troubleshooting of the system.

\section{T-S Fuzzy Fault Tree Importance Analysis}

\subsection{Fuzzy Number}

In order to solve the problem that the fault probability and fault severity can not be obtained accurately in the traditional fault tree analysis, the probability of occurrence and the degree of failure of the event are described by fuzzy number.

Define the fuzzy number, $\tilde{A}=(m, \alpha, \beta)$, where, $m$ is the mean of the fuzzy number $\tilde{A} ; \alpha, \beta$ are the upper and lower limit, when $\alpha, \beta$ are $0, \tilde{A}$ is a definite number, when $\alpha, \beta$ are bigger, $\tilde{A}$ is more blurry.

If the $\tilde{A}$ membership function satisfies: $\mu_{\tilde{A}}(x)= \begin{cases}1 & x=m \\ L(x) & x<m \\ R(x) & x>m\end{cases}$

Then $\tilde{A}$ is L-R type fuzzy number, $\mu_{\tilde{A}}^{(x)}$ is the membership function of $\tilde{A}$. The trapezoidal membership function shown in Fig. 1 to describe the fuzzy number $\tilde{A}$, the expression is: 


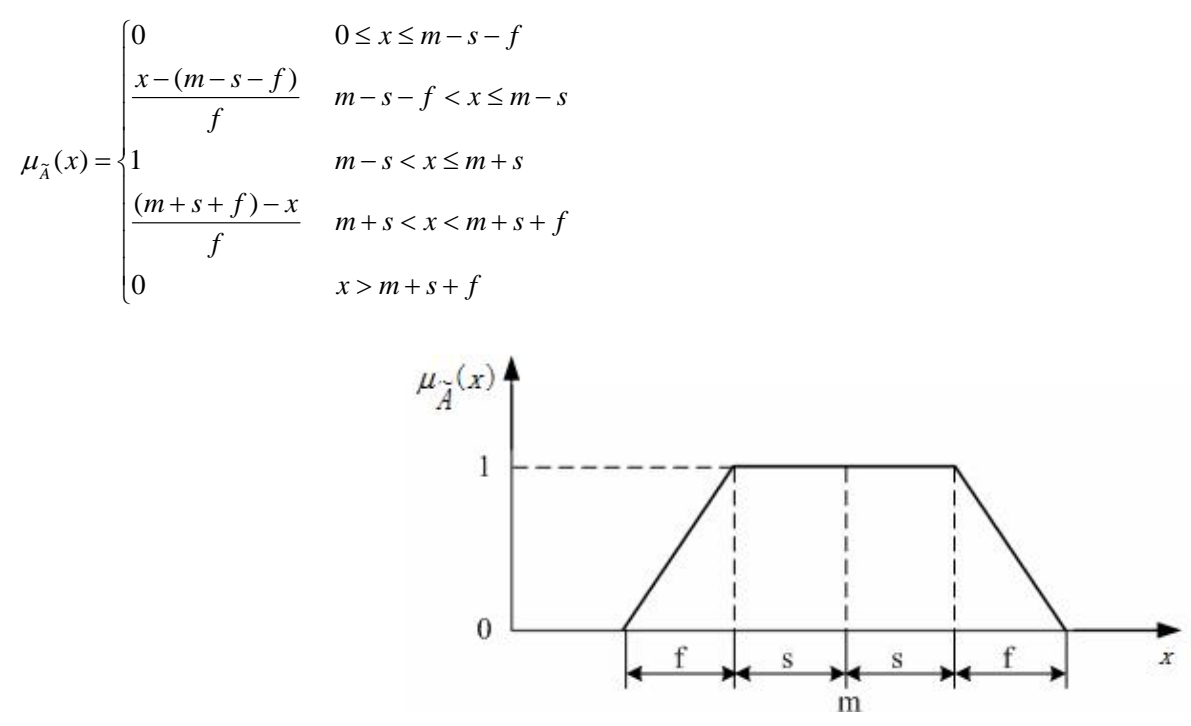

Fig.1 Trapezoidal Membership Function

Where, $\mathrm{m}$ is the center of the fuzzy suppori set, $\mathrm{s}$ is the support radius, $\mathrm{f}$ is the fuzzy area.The trapezoidal membership function is a versatile function, when $s=0$, the trapezoidal membership function becomes a triangular membership function; when $\mathrm{f}=0$, fuzzy number becomes determine number.

The important use of fuzzy numbers is used to describe the severity of the fault, which can be described by the number $[0,1]$ of fuzzy numbers on the interval, such as failure, minor failure, serious failure, etc., which can be described quantitatively with fuzzy numbers 0,0.5,1.

\subsection{T-S Gate Rule}

T-S model is consisted by a set of IF-THEN rules, if $x=\left\{x_{1}, x_{2}, \cdots, x_{n}\right\}$ is the front variable, $y$ is the latter variable, $A_{i j}(j=1,2, \cdots, n)$ is fuzzy sets, the expression is:

If fuzzy number $\left\{x_{1}^{(1)}, x_{1}^{(2)}, \cdots x_{1}^{\left(k_{1}\right)}\right\},\left\{x_{2}^{(1)}, x_{2}^{(2)}, \cdots x_{2}^{\left(k_{2}\right)}\right\}, \cdots,\left\{x_{n}^{(1)}, x_{n}^{(2)}, \cdots x_{n}^{\left(k_{n}\right)}\right\}$ and $\left\{y^{(1)}, y^{(2)}, \cdots, y^{\left(k_{y}\right)}\right\}$ used to describe the degree of failure of the front $x=\left\{x_{1}, x_{2}, \cdots, x_{n}\right\}$ and the latter $y$, when $0 \leq x_{1}^{(1)}<x_{1}^{(2)}<\cdots x_{1}^{\left(k_{1}\right)} \leq 1$, $0 \leq x_{2}^{(1)}<x_{2}^{(2)}<\cdots x_{2}^{\left(k_{2}\right)} \leq 1,0 \leq x_{n}^{(1)}<x_{n}^{(2)}<\cdots<x_{n}^{\left(k_{n}\right)} \leq 1,0 \leq y^{(1)}<y^{(2)}<\cdots<y^{\left(k_{2}\right)} \leq 1$, then the T-S gate algorithm can be expressed as follows:

Role $l$ : if $x_{1}$ is $x_{1}^{\left(i_{1}\right)}$, and $x_{2}$ is $x_{2}^{\left(i_{2}\right)}, \ldots$, and $x_{n}$ is $x_{n}^{\left(i_{n}\right)}, y$ is the possibility of $y^{(1)}$ is $P^{(l)}\left(y^{(1)}\right), y$ is the possibility of $y^{(2)}$ is $P^{(l)}\left(y^{(2)}\right), \cdots y$ is the possibility of $y^{\left(k_{y}\right)}$ is $P^{(l)}\left(y^{\left(k_{y}\right)}\right)$. Where, $i_{1}=1,2, \cdots, k_{1} ; i_{2}=1,2, \cdots, k_{2}$; $i_{n}=1,2, \cdots, k_{n}$. Then , the rule is $r=\prod_{i=1}^{n} k_{i}$.

Assuming that the blurring probability $P\left(x_{1}^{\left(i_{1}\right)}\right), P\left(x_{2}{ }^{\left(i_{2}\right)}\right), \cdots, P\left(x_{n}{ }^{\left(i_{n}\right)}\right)$ are used to describle the probability of occurrence of various failures at the bottom event, the probability of the rule $l$ execution is:

$$
P_{0}^{(l)}=P\left(x_{1}^{\left(i_{1}\right)}\right) P\left(x_{2}^{\left(i_{2}\right)}\right) \cdots P\left(x_{n}{ }^{\left(i_{n}\right)}\right)
$$

Therefore, the fuzzy possibility of the latter is:

$$
\left\{\begin{array}{c}
P\left(y^{(1)}\right)=\sum_{l=1}^{r} P_{0}^{(l)} P^{(l)}\left(y^{(1)}\right) \\
P\left(y^{(2)}\right)=\sum_{l=1}^{r} P_{0}^{(l)} P^{(l)}\left(y^{(2)}\right) \\
\vdots \\
P\left(y^{\left(k_{y}\right)}\right)=\sum_{l=1}^{r} P_{0}^{(l)} P^{(l)}\left(y^{\left(k_{y}\right)}\right)
\end{array}\right.
$$




\section{Research on Reliability of Pumping Hydraulic System}

An enterprise-made HBT60 concrete pump main pumping hydraulic system showed in Figure 2, and the main oil without pressure for the top event of the T-S fuzzy fault tree showed in Figure 3. The top event $T$ represents the pumping main system without pressure, the intermediate event $M$ represents, and the bottom event $x_{1}, x_{2}, x_{3}, x_{4}, x_{5}, x_{6}$ is represented separately.

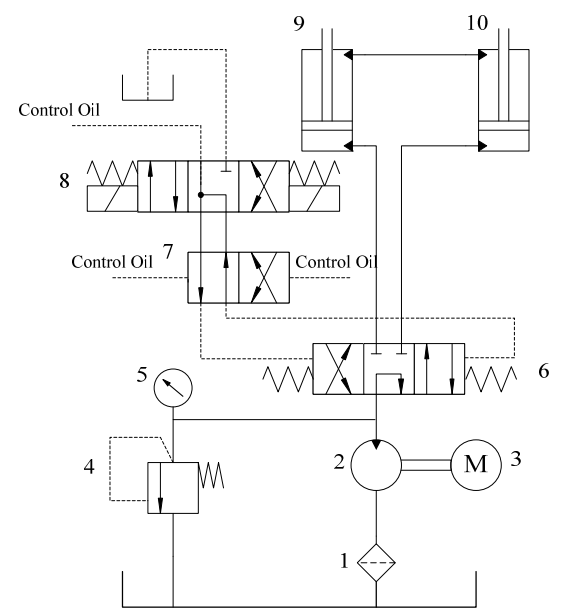

1-Suction filer;2-Main pump;3-Motor;

4- Pressure relief valve;5- pressure gauge;

6, 7-Hydraulic operated directional valve;

8--Solenoid valves;9, 10-Main cylinder;

Fig.2 Main pumping circuit

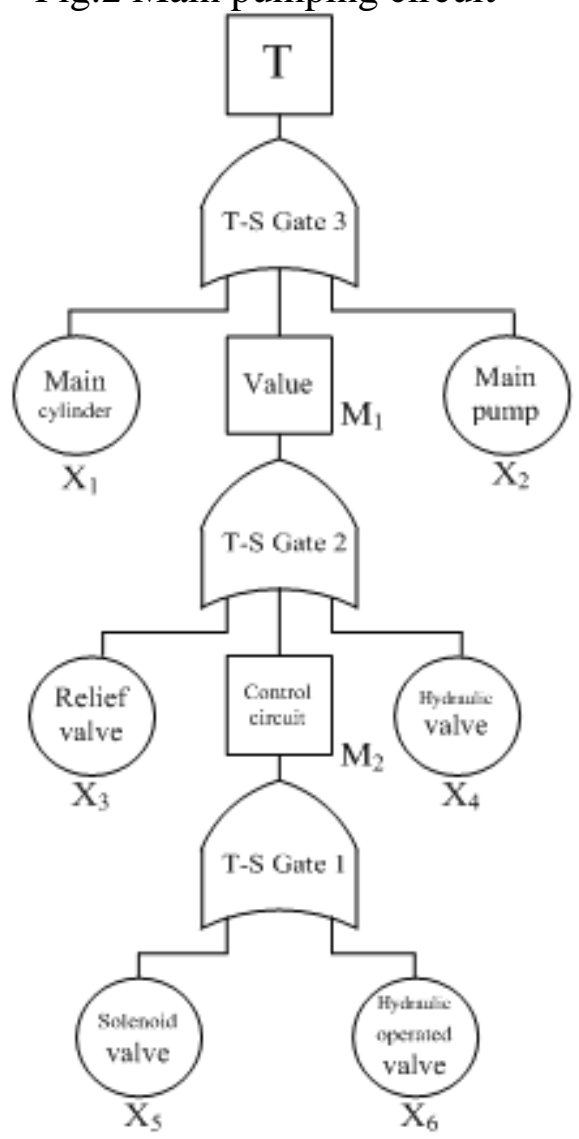

Fig.3 Main pumping circuit

Assume that the common fault degree of $x_{1}, x_{2}, x_{3}, x_{4}, x_{5}, x_{6}, T, M_{1}, M_{2}$ is $(0,0.5,1)$. Where 0 indicates no fault, that is, the pumping system pressure flow is normal, the hydraulic system can complete the pumping function; 0.5 indicates the semi-fault state or the mild fault condition, that is, the pressure 
flow instability is not reach the specified value, the system can not be completed Specify the function; 1 that a complete failure or serious fault, that is, pressure flow is almost zero, the system can not work. In conjunction with the trapezoidal membership function shown in Figure 2, the parameters are selected as $s=0.1, f=0.3$. Based on expert experience, three T-S door rules are listed.

Table 1. T-S Gate 1 Rule

\begin{tabular}{cccccc}
\hline & & & & $M_{2}$ & \\
\cline { 3 - 6 } Rule & $x_{5}$ & $x_{6}$ & 0 & 0.5 & 1 \\
\hline 1 & 0 & 0 & 1 & 0 & 0 \\
2 & 0 & 0.5 & 0.2 & 0.6 & 0.2 \\
3 & 0 & 1 & 0 & 0 & 1 \\
4 & 0.5 & 0 & 0.2 & 0.6 & 0.2 \\
5 & 0.5 & 0.5 & 0.2 & 0.2 & 0.6 \\
6 & 0.5 & 1 & 0 & 0 & 1 \\
7 & 1 & 0 & 0 & 0 & 1 \\
8 & 1 & 0.5 & 0 & 0 & 1 \\
9 & 1 & 1 & 0 & 0 & 1 \\
\hline
\end{tabular}

Table 2. T-S Gate 2 Rule

\begin{tabular}{|c|c|c|c|c|c|c|}
\hline \multirow{2}{*}{ Rule } & \multirow{2}{*}{$M_{2}$} & \multirow{2}{*}{$x_{3}$} & \multirow{2}{*}{$X_{4}$} & \multicolumn{3}{|c|}{$M_{1}$} \\
\hline & & & & 0 & 0.5 & 1 \\
\hline 1 & 0 & 0 & 0 & 1 & 0 & 0 \\
\hline 2 & 0 & 0 & 0.5 & 0.2 & 0.6 & 0.2 \\
\hline 3 & 0 & 0 & 1 & 0 & 0 & 1 \\
\hline 4 & 0 & 0.5 & 0 & 0.2 & 0.6 & 0.2 \\
\hline 5 & 0 & 0.5 & 0.5 & 0.2 & 0.2 & 0.6 \\
\hline 6 & 0 & 0.5 & 1 & 0 & 0 & 1 \\
\hline 7 & 0 & 1 & 0 & 0 & 0 & 1 \\
\hline 8 & 0 & 1 & 0.5 & 0 & 0 & 1 \\
\hline 9 & 0 & 1 & 1 & 0 & 0 & 1 \\
\hline --- & --- & --- & --- & --- & --- & --- \\
\hline 27 & 1 & 1 & 1 & 0 & 0 & 1 \\
\hline \multicolumn{7}{|c|}{ Table 3 T-S Gate 3 Rul } \\
\hline \multirow{2}{*}{ Rule } & \multirow{2}{*}{$M_{1}$} & \multirow{2}{*}{$X_{1}$} & \multirow[b]{2}{*}{$x_{2}$} & \multicolumn{3}{|c|}{$T$} \\
\hline & & & & 0 & 0.5 & 1 \\
\hline 1 & 0 & 0 & 0 & 1 & 0 & 0 \\
\hline 2 & 0 & 0 & 0.5 & 0.2 & 0.6 & 0.2 \\
\hline 3 & 0 & 0 & 1 & 0 & 0 & 1 \\
\hline 4 & 0 & 0.5 & 0 & 0.2 & 0.6 & 0.2 \\
\hline 5 & 0 & 0.5 & 0.5 & 0.2 & 0.2 & 0.6 \\
\hline 6 & 0 & 0.5 & 1 & 0 & 0 & 1 \\
\hline 7 & 0 & 1 & 0 & 0 & 0 & 1 \\
\hline 8 & 0 & 1 & 0.5 & 0 & 0 & 1 \\
\hline 9 & 0 & 1 & 1 & 0 & 0 & 1 \\
\hline--- & --- & --- & --- & --- & --- & --- \\
\hline 27 & 1 & 1 & 1 & 0 & 0 & 1 \\
\hline
\end{tabular}


Table 4. Bottom Event Failure Fuzzy Probability

\begin{tabular}{ccc}
\hline Event & Fuzzy Probability Mean & Trapezoidal Fuzzy Number \\
\hline x1 & 0.057 & $(0.051,0.055,0.059,0.063)$ \\
x2 & 0.0067 & $(0.0007,0.0047,0.0087,0.0127)$ \\
x3 & 0.0142 & $(0.0082,0.0122,0.0162,0.0202)$ \\
x4 & 0.0192 & $(0.0132,0.0172,0.0212,0.0252)$ \\
x5 & 0.0017 & $(0.0011,0.0015,0.0019,0.0023)$ \\
x6 & 0.0017 & $(0.0011,0.0015,0.0019,0.0023)$ \\
\hline
\end{tabular}

A concrete pump manufacturer provides the failure probability data for the bottom event, as shown in Table 4 after blurring.

The probability of failure in Table 4 is the sum of the failure degree of 0.5 at each end event $x_{1}, x_{2}, x_{3}, x_{4}, x_{5}, x_{6}$ and the degree of failure of 1 . Assume that the probability of occurrence of two different levels of failure is the same. According to the rules of T-S gate 1 in Table 1, the fault probability data of X in Table 4,and formula (2), (3), the probability of failure degree of intermediate event $\mathrm{M}$ of 0.5 and 1 is:

$\mathrm{P}\left\{\mathrm{M}_{2}=0.5\right\}=\sum_{i=1}^{9} P_{0}^{l} P^{l}\left(M_{2}=0.5\right)=P_{0}^{2} P^{2}\left(M_{2}=0.5\right)+P_{0}^{4} P^{4}\left(M_{2}=0.5\right)+P_{0}^{5} P^{5}\left(M_{2}=0.5\right)$ $=0.6 \times P_{0}^{2}+0.6 \times P_{0}^{4}+0.2 \times P_{0}^{5}=(0.00132,0.0018,0.00228,0.00276)$ $\mathrm{P}\left\{\mathrm{M}_{2}=1\right\}=\sum_{i=1}^{9} P_{0}^{l} P^{l}\left(M_{2}=1\right)=P_{0}^{2} P^{2}\left(M_{2}=1\right)+P_{0}^{3} P^{3}\left(M_{2}=1\right)+P_{0}^{4} P^{4}\left(M_{2}=1\right)+P_{0}^{5} P^{5}\left(M_{2}=\right.$ 1) $+P_{0}^{6} P^{6}\left(M_{2}=1\right)+P_{0}^{7} P^{7}\left(M_{2}=1\right)+P_{0}^{8} P^{8}\left(M_{2}=1\right)+P_{0}^{9} P^{9}\left(M_{2}=1\right)=0.2 \times P_{0}^{2}+1 \times P_{0}^{3}+0.2 \times$ $P_{0}^{4}+0.6 \times P_{0}^{5}+1 \times P_{0}^{6}+1 \times P_{0}^{7}+1 \times P_{0}^{8}+1 \times P_{0}^{9}=(0.00264,0.0036,0.00456,0.00552)$

It can be seen that the probability of the intermediate event $M_{2}$ failure degree of 0.5 and 1 is still a trapezoidal fuzzy number which is of the same order of magnitude as the failure probability of the bottom event ${ }_{5}, x_{6}$ and the probability that the intermediate event $M_{2}$ has a fault level of 1 is greater than the failure of the bottom event $x_{5}, x_{6}$ Probability, which is consistent with the actual situation.

Using the same method, the probability of finding the top event failure level of 0.5 and 1 is:

$\mathrm{P}\{T=0.5\}=(0.0023,0.027,0.031,0.035)$

$\mathrm{P}\{T=1\}=(0.139,0.169,0.199,0.229)$

The results are in good agreement with the failure probability of the main oil in the main hydraulic system of the concrete pump pumped hydraulic system, which verifies the correctness of the proposed T-S fuzzy fault tree algorithm.

\section{Results and Discussion}

Based on the combination of TS fuzzy model and fault tree analysis, a method of reliability analysis of hydraulic system based on the importance degree of T-S fuzzy fault tree is proposed. This method used fuzzy number to describe the fault probability of event. The fault probability is solved by T-S fuzzy gate instead of the connection between the fault description and the event in the traditional fault tree. The fuzzy number is used to describe the fault of the fault and the fault of the event. The paper analyzed the influence of the fault tree analysis, and analyzes the characteristics of the fault of the hydraulic system. The method is based on the T-S fuzzy model which can be infinitely approximated to the real system. In this paper, dealing with fuzzy information and fault mechanism uncertainty reasoning more flexibility and accuracy.

\section{Acknowledgements}

In this paper, the research was sponsored by the Scientific Research Fund of Hunan Provincial Education Department (Project No. 14C1162) and Youth Science Research Foundation of Central South University of Forestry and Technology (Project No. QJ201509). 


\section{References}

[1]. Avontuur G C,van der Werff Systems Reliability Analysis of Mechanical and Hydraulic Drive Systems[J].Reliability Engineering and System Safety,2002,77 (2):121-130.

[2]. Zhao Jing-yi,Yao Cheng-yu.Hydraulic System Reliability Engineering[M], Beijing: Mechanical Industry Press, 2011.

[3]. Yao C Y,Zhao J Y.Research on Fuzzy Tree Analysis Method for Hydraulic System Based on TS Model[J], Chinese Journal of Mechanical Engineering,2009, 16(20): 1913-1917.

[4]. Tang Hong-bin,WU Yun-xin.Fault Diagnosis of Hydraulic System of Concrete Pump Truck Based on T-S Fuzzy Fault Tree Analysis[J], Chinese Hydraulics and Pneumatics.2009, (10):6365. 